\title{
Las exposiciones interactivas para escolares como herramienta de motivación de los estudiantes de Ingeniería Forestal y del Medio Natural
}

\author{
E. García ${ }^{1}$, J.R. Villar ${ }^{1}$ y M. Moya ${ }^{1}$ \\ 1 Universidad de Extremadura. Centro Universitario de Plasencia. Avda. Virgen del Puerto no 2, 10600 \\ Plasencia (Cáceres); egciadel@unex.es; jrvillar@unex.es; manuelmi@unex.es
}

\begin{abstract}
Resumen: La innovación docente se concibe como la vía idónea para mejorar la formación del alumnado en competencias técnicas y transversales en el Grado en Ingeniería Forestal y del Medio Natural de la Universidad de Extremadura, pues los estudiantes presentan importantes carencias, especialmente de tipo técnico, al haber accedido al Grado, mayoritariamente, desde algún ciclo formativo de grado superior. Estas carencias reducen sensiblemente su motivación para el estudio y para la adquisición de dichas competencias. Para contrarrestar este problema se ha diseñado una estrategia innovadora bajo el lema "FOREXPO: enseñando se aprende más", consistente en una exposición interactiva sobre espacios naturales protegidos, física y medio ambiente, que es ofrecida por los estudiantes del Grado a los escolares de la ciudad de Plasencia cada primavera, desde 2013, para aprender de manera directa y amena a través de maquetas y exposiciones de tipo interactivo en las que estos últimos pueden experimentar, tocar, observar, etc. FOREXPO es novedoso porque combina diversas metodologías innovadoras, como el Aprendizaje Basado en Problemas (ABP), el Aprendizaje por Servicio (ApS), el "enseñando se aprende más" (learning by teaching) y porque, además, facilita la colaboración entre dos materias del Grado, algo muy enriquecedor desde muchos puntos de vista. Finalmente, con esta actividad se contribuye a difundir la labor del Ingeniero Forestal en la sociedad combinando tanto la parte científica como la parte medioambiental de manera educativa.
\end{abstract}

Palabras clave: FOREXPO, innovación docente, Aprendizaje Basado en Problemas, Aprendizaje por Servicio, enseñando se aprende más

\section{Introducción}

El Grado en Ingeniería Forestal y del Medio Natural (IFMN) de la Universidad de Extremadura (UEX), ofertado en el Centro Universitario de Plasencia, se caracteriza porque en los últimos años un porcentaje elevado de los alumnos de nuevo ingreso proceden de ciclos formativos de grado superior gracias a la aprobación del Real Decreto 1618/2011, de 14 de noviembre, sobre reconocimiento de estudios en el ámbito de la educación superior (BOE núm. 302, de 16 de diciembre de 2011). En la formación que recibe este tipo de alumnado se detecta una carencia importante en materias de base técnica, que son esenciales en los Grados de Ingeniería, lo que conlleva el que la mayoría de estos estudiantes pierdan la motivación para el estudio y, por ende, también para la adquisición de competencias transversales que son cada vez más demandadas en el ámbito profesional.

Como se definía en el proyecto "Tunning Educational Structures in Europe", "las competencias representan una combinación dinámica de atributos en relación con conocimientos, habilidades, actitudes y responsabilidades, que describen los resultados de aprendizaje de un 


\section{CONGRESO IBÉRICO DE AGROINGENIERÍA \\ X CONGRESSO IBÉRICO DE AGROENGENHARIA \\ 3 - 6 septiembre 2019, Huesca - España}

programa educativo o lo que los alumnos son capaces de demostrar al final de un proceso educativo" [1]. Con esta definición la competencia implica la movilización de distintos atributos del alumno, tanto visibles (conocimientos y habilidades), como invisibles (actitudes y responsabilidades).

Por este motivo, desde la implantación del proceso de Bolonia en la universidad española, el profesorado del Grado en IFMN de la UEX se ha preocupado por la innovación docente, como lo atestigua la concesión y posterior desarrollo de diversos proyectos competitivos de este tipo para lograr fortalecer las carencias detectadas en el alumnado, tanto técnicas como transversales [2-5], mejorando a la vez tanto las tasas de éxito alcanzadas como el rendimiento en las materias implicadas.

Entre las competencias transversales cabe destacar la importancia creciente que posee la inteligencia emocional. Este concepto, que es relativamente nuevo, fue utilizado por primera vez por Peter Salovey y John Mayer en 1990 [6], y se ha hecho tremendamente popular porque explica y evidencia cómo personas con un buen cociente intelectual fracasan en ocasiones $\mathrm{y}$, sin embargo, otras que poseen un cociente intelectual más bajo fracasan escolarmente pero posteriormente triunfan en diversos ámbitos [7]. Este mismo autor [8] propuso un modelo de inteligencia emocional sustentado en cuatro brazos (autoconciencia, autogestión, conciencia social y gestión de relaciones), de cada uno de los cuales posteriormente obtenía diversas competencias emocionales, tales como la autoconciencia emocional, la autoconfianza, el autocontrol, la integridad, la adaptabilidad, la iniciativa, la empatía, la comunicación, la gestión de conflictos o el liderazgo, entre otros, considerando un total de 20 competencias. Estas competencias se han ido trasladando posteriormente al mundo profesional y a los negocios, de modo que lo utilizan muchas empresas actualmente para tratar de encontrar los mejores candidatos para determinados puestos de trabajo que puedan requerir en un momento dado.

Para lograr que los estudiantes de la titulación sean capaces de desarrollar las competencias técnicas y transversales deseadas en su formación, las metodologías docentes elegidas deben ser las más adecuadas para ello, centrándose fundamentalmente en el papel activo del alumno en lugar de su papel pasivo, como se viene haciendo en la enseñanza tradicional [9]. De entre las existentes, las del "Aprendizaje Basado en Problemas" (ABP), impulsada, entre otros, por Olsen y Pedersen [10], el Aprendizaje por Servicio (ApS), que se desea arraigar en el ámbito universitario [11] y la de "Enseñando se aprende más" (learning by teaching), de la que también se han realizado experiencias piloto y se intenta implantar su uso con fines pedagógicos [12-15], son las elegidas por los profesores implicados en la actividad "FOREXPO: enseñando se aprende más" porque se han considerado idóneas para el alumnado del Grado en IFMN. Con ella se espera poder lograr en los estudiantes resultados parecidos a los alcanzados en otras experiencias en las que los alumnos enseñan a otros alumnos [16-18], además de facilitar la colaboración entre dos materias del Grado, algo muy enriquecedor desde muchos puntos de vista tanto para los profesores como para los alumnos que participan en su desarrollo.

Finalmente, con esta actividad se contribuye a difundir la labor del Ingeniero Forestal en la sociedad combinando tanto la parte científica como la parte medioambiental de manera educativa.

\section{FOREXPO: Enseñando se aprende más}

La actividad "FOREXPO: enseñando se aprende más" se viene proponiendo desde el curso 2012/2013 en el Grado en IFMN de la UEX en el Centro Universitario de Plasencia. Inicialmente se promovió desde la asignatura de "Física", asignatura obligatoria de primer curso del Grado, pero posteriormente, desde el curso 2014/15, se sumó a esta propuesta la asignatura "Gestión de Espacios Naturales Protegidos", optativa para alumnos de tercer y cuarto curso de la titulación. Desde entonces, y hasta la actualidad, ya han sido cinco las ediciones celebradas de esta actividad y los resultados obtenidos no pueden ser más satisfactorios, como se verá posteriormente al 


\section{CONGRESO IBÉRICO DE AGROINGENIERÍA \\ X CONGRESSO IBÉRICO DE AGROENGENHARIA \\ 3 - 6 septiembre 2019, Huesca - España}

analizar los resultados de las encuestas que se han realizado entre los participantes en la última edición celebrada correspondiente al curso 2018/19.

La asignatura de "Física" suele ser complicada para la mayoría de los estudiantes del Grado en IFMN porque, como se comentó en la sección anterior, muchos de ellos no tienen base técnica suficiente al provenir de ciclos formativos de grado superior. Este hecho provocó el que, tras la implantación del proceso de Bolonia en la UEX, al comenzar a recabarse datos sobre las tasas de éxito de las distintas materias impartidas, en esta asignatura concretamente dicha tasa fuera una de las más bajas de la titulación, y todo ello a pesar del enorme esfuerzo realizado por la profesora responsable de la asignatura para suplir las carencias de base que ya por aquel entonces se habían detectado. Por este motivo, para tratar de reconducir la situación, se percibió que los alumnos debían adoptar un papel más activo en la asignatura, dejando de ser meros receptores de información. Para ello, se plantearon diversos talleres de física que los propios alumnos debían ser capaces de poder desarrollar y explicar posteriormente y con los que se pretendía que aprendieran de manera más interactiva e intuitiva algunos de los fundamentos de la física cuya explicación teórica ya se les había facilitado previamente en clase, comprobando de primera manera mano cómo se cumplen en la realidad en la vida cotidiana.

Sin embargo, no sólo se plantea esta actividad únicamente desde el punto de vista de comprender mediante su puesta en práctica algunos de los fundamentos de la física, sino que también se desea ayudarles en el desarrollo de las competencias transversales, tan demandadas en el ámbito profesional actualmente. Al constatar que muchos de los estudiantes de los últimos cursos tenían muchas dificultades en ese sentido, por ejemplo, al defender públicamente sus trabajos fin de grado, pues les cuesta bastante hablar en público expresándose correctamente, o a organizarse para sacar delante de manera exitosa trabajos en grupo que se requieren en algunas asignaturas, entre otros aspectos, se creyó conveniente que los estudiantes comenzaran cuanto antes a desarrollar este otro tipo de habilidades. Por eso, se concibió que una buena manera de desarrollar conjuntamente estas competencias era conformar pequeños grupos para preparar y desarrollar cada uno de los talleres previstos, así como obligarles a exponer éstos en público, poniendo así en juego las metodologías de Aprendizaje Basado en Problemas y "enseñando se aprende más".

Como receptores de estos talleres se escogió a escolares que cursan tercero, cuarto, quinto o sexto de educación primaria. A esa edad los escolares están en condiciones idóneas para despertar en ellos el interés por la ciencia y el respeto por el medio ambiente. Por eso, aprovechando que esa labor de educación y sensibilización se viene haciendo por parte del profesorado de educación primaria, qué mejor manera de refrendarlo que permitir que ese mismo mensaje les llegue desde estudiantes universitarios que, con ejemplos prácticos muy sencillos de realizar, les permiten comprobar cómo aquello que se les explica en las aulas se lleva a cabo en la realidad. Pero es que, además, se les permite tocar lo que quieran, jugar con parte del material que se utiliza en estos talleres, observar, analizar, deducir y, en definitiva, participar activamente en una visita a la Universidad que seguro que no olvidarán fácilmente con el paso de los años. Se pone aquí en práctica, por lo tanto, la metodología del "Aprendizaje por servicio".

Dada la buena acogida que tuvo esta actividad en sus primeros años, se decidió que desde la asignatura "Gestión de Espacios Naturales Protegidos" también se propusiera algún taller, en este caso dándole un enfoque completamente medioambiental, sobre alguna de las temáticas que se tratan en la asignatura, aprovechando la excelente relación que existe entre los profesores responsables de ambas asignaturas. En este caso, al tratarse de una asignatura de carácter optativo, el número de alumnos que se matriculan en ella es menor, pues hay otras nueve asignaturas optativas a elegir y de ellas deben seleccionar cinco para finalizar la carrera, por lo que en este caso se propone un único taller pero con los mismos requisitos que se exigen en física, es decir, que se realice y se exponga en grupo ante los escolares que visitan el Centro Universitario de Plasencia. 


\section{CONGRESO IBÉRICO DE AGROINGENIERÍA \\ X CONGRESSO IBÉRICO DE AGROENGENHARIA \\ 3- 6 septiembre 2019, Huesca - España}

Además, dado que en Plasencia mucha gente desconoce aún lo que se hace en el Centro Universitario, incluso muchos desconocen que hay un Centro Universitario, pues éste se asienta en un edificio que era un antiguo cuartel y que fue rehabilitado para convertirlo en un centro educativo (motivo por el que muchos placentinos aún denominan al edificio el "antiguo cuartel"), con el fin de difundir la titulación y acercar la ciencia a la sociedad, se dio difusión de esta actividad a los colegios de Plasencia y de otros municipios cercanos para que trajesen a sus alumnos a dicho Centro para que éstos disfrutaran y aprendieran con los talleres desarrollados por nuestros estudiantes. Por cuestiones de agenda, pues a los estudiantes se les computa las horas de trabajo totales que deben dedicarles a las distintas asignaturas, fijándose éstas en 150 horas en todo el cuatrimestre (6 créditos ECTS para cada asignatura), a esta actividad sólo se le puede dedicar entre tres y cuatro sesiones, celebrándose tradicionalmente los viernes por la mañana, pues es buen momento para los escolares, y preferiblemente en el mes de abril, porque para entonces los estudiantes han tenido tiempo desde febrero (mes en el que comienza el segundo cuatrimestre) para ir familiarizándose con ambas asignaturas, aprender algunos de los conceptos y fundamentos de las mismas, y poder organizarse para preparar los distintos talleres que expondrán posteriormente.

La duración de los talleres es de 15 minutos, de modo que a los colegios se les pide que compongan grupos de 50 escolares, aproximadamente, albergando a dos grupos cada una de las mañanas (unos 100 alumnos en total en cada una de las sesiones). Cada uno de estos grupos se divide en tres para los talleres de física, conformando subgrupos de 15-16 estudiantes para cada uno de los talleres que se les explica después, aproximadamente, mientras que para el taller de espacios naturales protegidos se permite que permanezcan los 50 juntos. Por este motivo, los estudiantes de física exponen 6 talleres a lo largo de cada una de las mañanas en las que se desarrolla FOREXPO, en los que se explican fenómenos como el funcionamiento de la capa freática, la sostenibilidad de las construcciones de paja, el comportamiento de los fluidos no newtonianos, el motor Stirling o el funcionamiento de un coche de hidrógeno, entre otros, mientras que los estudiantes de gestión de espacios naturales protegidos sólo desarrollan dos talleres cada vez (el mismo repetido), eligiendo temáticas diversas como la necesidad de creación de espacios naturales protegidos, los tipos de áreas protegidas que existen, las peculiaridades del Parque Nacional de Monfragüe, las especies más representativas de este espacio o pautas de buena conducta que se han de llevar a cabo en este tipo de espacios. Las figuras 1 y 2 ilustran el desarrollo de estos talleres.
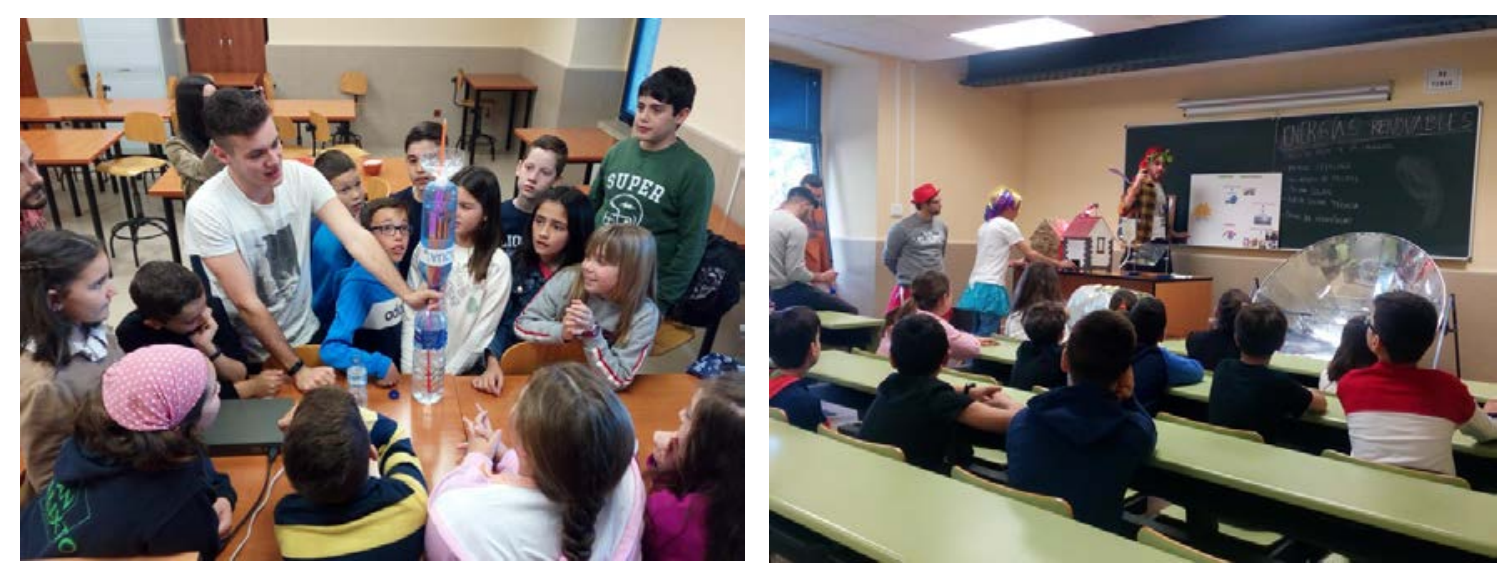

Figura 1. Talleres de física expuestos por los alumnos de primer curso del Grado en Ingeniería Forestal y del Medio Natural de la UEX. 

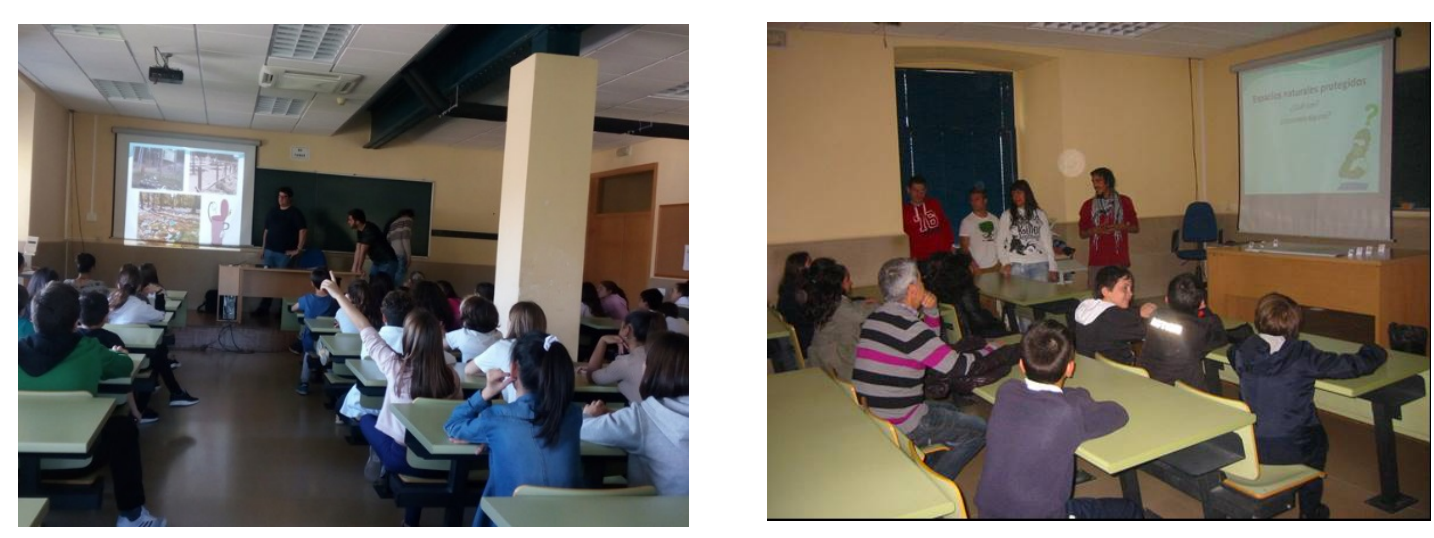

Figura 2. Talleres de gestión de espacios naturales protegidos expuestos por los alumnos de tercer y cuarto curso del Grado en Ingeniería Forestal y del Medio Natural de la UEX.

Conviene aclarar, no obstante, que los alumnos de física deben familiarizarse con todos los talleres que se proponen porque van rotando de unas semanas a otras, de modo que al final acaban comprendiendo todos los talleres planteados porque tienen que exponerlos ante los escolares, mientras que a los alumnos de gestión se les cita un único día (si hay alumnos suficientes matriculados en la asignatura), dos como mucho, pues la asignatura se imparte los miércoles y los jueves, de modo que los viernes están fuera del horario previsto para ella y se solapa en ocasiones con otras asignaturas que cursan algunos de los matriculados, por lo que se trata de facilitar su participación pero sin que ésta les impida compaginar esta actividad con las demás asignaturas.

\section{Difusión de FOREXPO}

Dado que en los últimos años se exige a los organismos públicos que rindan cuentas ante la sociedad para que ésta conozca qué se está haciendo, en el ámbito universitario la mejor manera de justificarse ante ella es dando a conocer por diversos medios las actividades desarrolladas y a las que el profesorado implicado dedica un gran esfuerzo y dedicación que muchas veces se desconoce fuera de este ámbito. Por esta razón, casi desde el principio, se han enviado notas de prensa para que los medios de comunicación se hicieran eco de esta actividad desarrollada en el Centro Universitario de Plasencia. Por este motivo, no es de extrañar que se hayan concedido algunas entrevistas en diversas cadenas de radio de ámbito local y/o regional y que, a su vez, la prensa escrita haya reflejado esta actividad en sus páginas ilustrándola debidamente. Se persigue así, por lo tanto, que la sociedad placentina esté al corriente de esta iniciativa, dando a conocer tanto la titulación como el Centro Universitario, para que cale en la sociedad la existencia de un centro educativo de estas características y un grado en el que se puede aprender enseñando a los demás. La figura 3 muestra algunas de las noticias aparecidas en los medios con motivo de la realización de FOREXPO en esta primavera de 2019. 


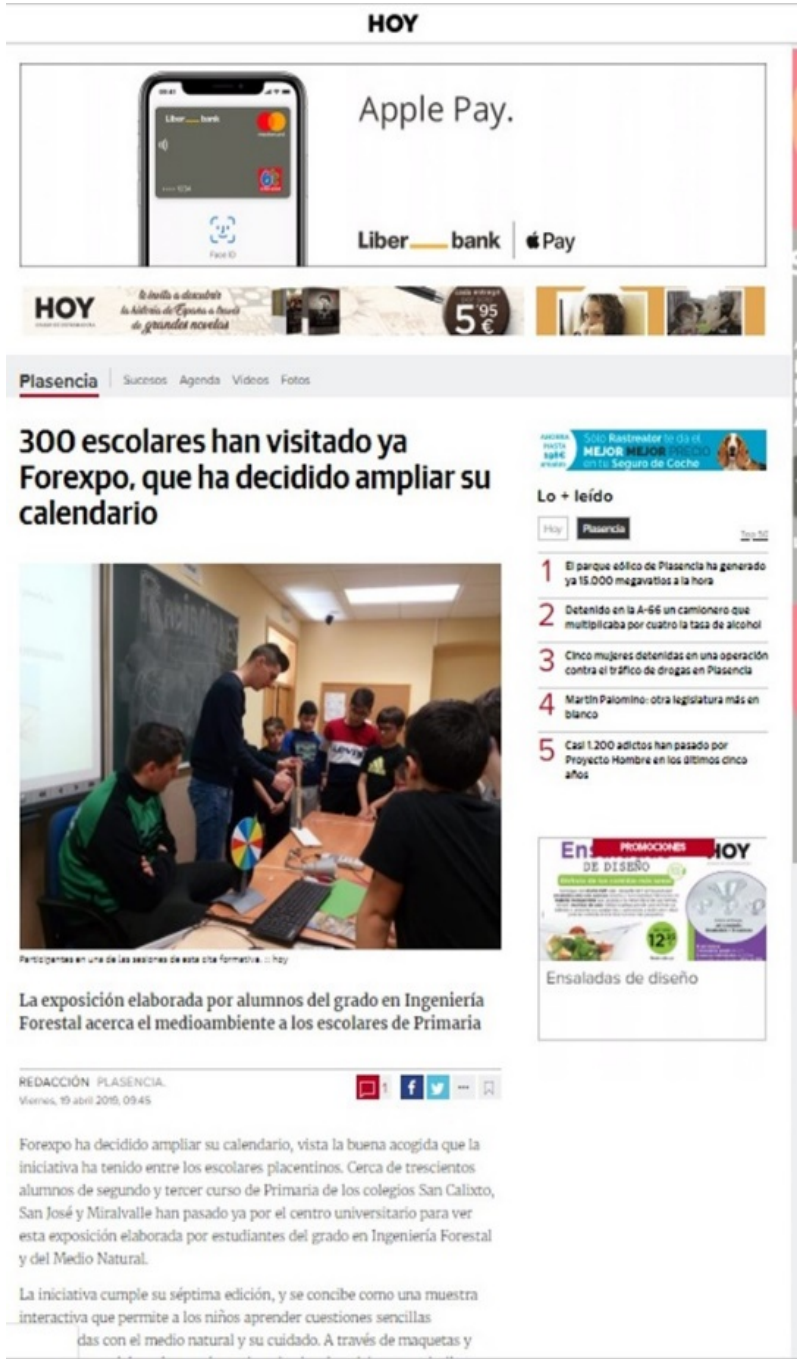

EXPOSICIÓN PARA ESCOLARES, EN LA UEX

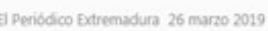

Viernes 22 y 29 de marzo y 5 de tbril, por séptimo ano consecutivo, os alumnos universitarios del Grado en Ingeniería Forestal del centro miversitario de la UEx confecciona vexplican Forexpo, una exposición nteractiva sobre Fisica, Medio Ambiente y Gestión de Espacios Natuales Protegidos. Los alumnos de segundo y tercer ciclo de Pirmaria le los colegios son los destinatarios. El objetivo es transmitir a los escoares la preocupación por el medio latural y por la adecuada gestión de os recursos.

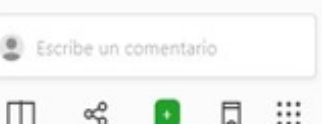

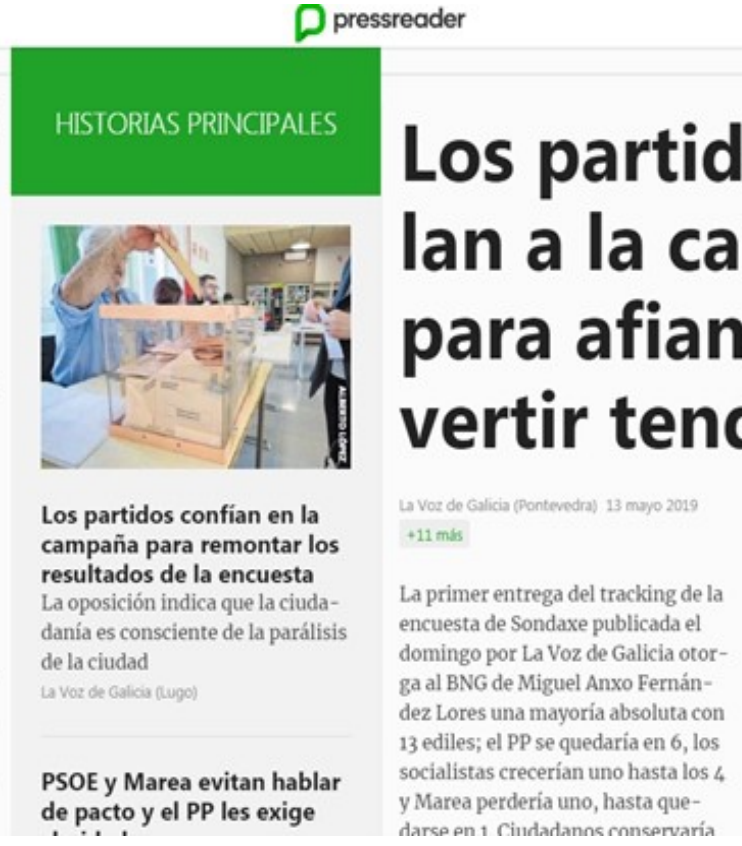

Figura 3. Noticias de FOREXPO reflejadas en periódicos de difusión regional. 


\section{CONGRESO IBÉRICO DE AGROINGENIERÍA \\ X CONGRESSO IBÉRICO DE AGROENGENHARIA \\ 3- 6 septiembre 2019, Huesca - España}

Pero no sólo se hacen eco del evento los medios de comunicación. Las figuras 4 y 5 muestran ejemplos de la difusión que dan los colegios que han tenido la oportunidad de llevar a sus alumnos al Centro Universitario de Plasencia para disfrutar de las jornadas de FOREXPO sobre lo acontecido en dicha actividad.

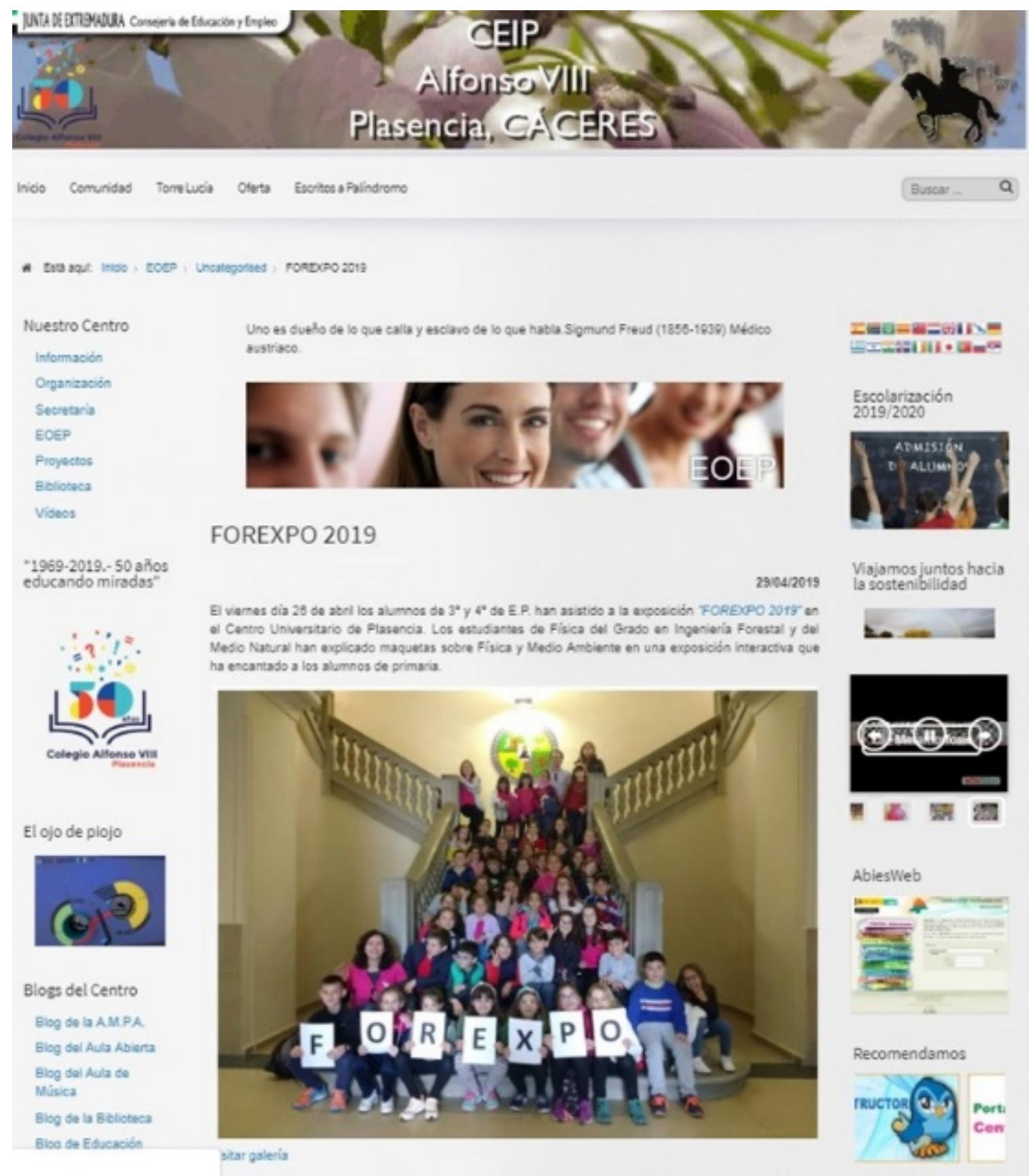

Figura 4. Difusión de FOREXPO en las páginas web de uno de los colegios de Plasencia que han visitado el Centro Universitario en el mes de abril de 2019. 


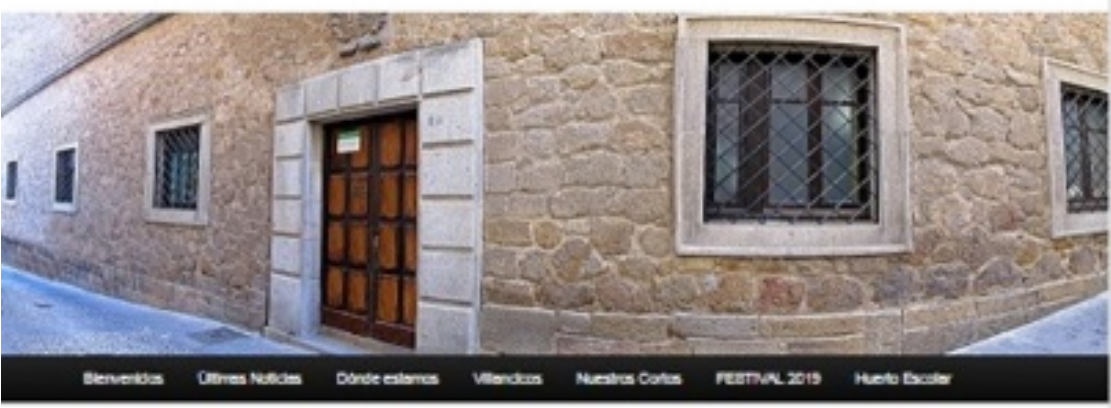

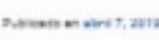

\section{FOREXPO 2019}

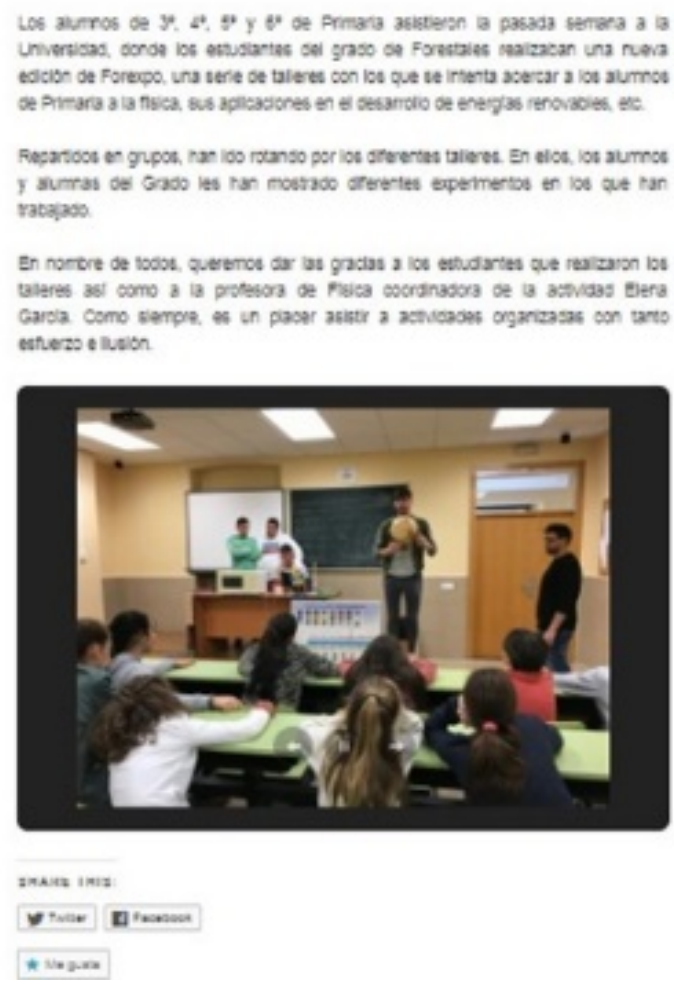

Figura 5. Difusión de FOREXPO en las páginas web de uno de los colegios de Plasencia que han visitado el Centro Universitario en el mes de abril de 2019.

\section{Resultados de las encuestas}

En la edición celebrada el pasado mes de abril de 2019 se ha realizado por primera vez una encuesta para conocer la opinión de los protagonistas que hacen posible la realización de "FOREXPO: enseñando se aprende más", los estudiantes del Grado en IFMN de la UEX, por un lado, y los profesores de los Centros de Educación Primaria que se han acercado al Centro Universitario con sus alumnos para disfrutar y participar en los talleres de Física y Gestión de Espacios Naturales Protegidos especialmente diseñados para ellos. El número de encuestas recogidas ha sido de 15 entre los alumnos de física, de primer curso del Grado en IFMN, que corresponden al $100 \%$ de los alumnos matriculados por primera vez en la asignatura, mientras que en el caso de la asignatura de Gestión de Espacios Naturales Protegidos sólo se han podido 


\section{CONGRESO IBÉRICO DE AGROINGENIERÍA \\ X CONGRESSO IBÉRICO DE AGROENGENHARIA \\ 3 - 6 septiembre 2019, Huesca - España}

recoger 4 encuestas de un total de 8 alumnos matriculados en la misma, pues los cuatro restantes no respondieron a la petición realizada para recabar su opinión sobre esta actividad. Por su parte, en lo que a la participación del profesorado visitante se refiere, en total fueron 5 las respuestas recogidas procedentes de dos de los colegios que visitaron el Centro. A pesar de no ser una cifra especialmente elevada, sí se valora muy positivamente su opinión porque refleja bastante bien el sentir del resto de profesores visitantes que se acercaron a FOREXPO pero de los que, desgraciadamente, no ha sido posible obtener respuesta en el momento de presentar este trabajo. Seguidamente se van a mostrar y comentar los resultados más destacados que se han obtenido desglosando éstos por bloques temáticos, pues así fue como se estructuró la encuesta preparada expresamente para tal fin.

En las cuestiones en las que se pedía que dieran una puntuación, los valores asignados para éstas oscilaban entre 1 y 5 . Así, 1 significaba que no están nada de acuerdo, 2 que están poco de acuerdo, 3 que les parece regular, 4 que están de acuerdo y 5 que están muy de acuerdo.

\subsection{Encuestas de los estudiantes del Grado en Ingeniería Forestal y del Medio Natural}

Con el primer bloque de preguntas se pretende conocer la impresión general de los estudiantes sobre la experiencia de participar en FOREXPO. En este apartado se les ha preguntado sobre la novedad, el interés, la carga horaria (entendiendo ésta como la suma del tiempo dedicado a la preparación de los talleres y el de la exposición en sí), el interés despertado en los asistentes, la coordinación con los compañeros y la organización. Cada uno de estos aspectos debían valorarlos de 1 a 5 . Los resultados más destacados que se han obtenido para este primer bloque de preguntas han sido los siguientes

La novedad es muy bien valorada, tanto por los alumnos de primero como por los de la optativa de GENP: aunque estos últimos ya experimentaron la iniciativa de FOREXPO durante su primer curso, les sigue pareciendo algo muy novedoso, pero en menor grado, pues el $70 \%$ asigna una puntación de 4 , mientras que entre los de primero se valoran con un 5 por parte de un porcentaje muy similar (un $67 \%$ ).

Por otra parte, a todos los alumnos les parece muy interesante la experiencia de aprender enseñando: para los de primero el interés se reparte mayoritariamente por igual entre un valor de $4(40 \%)$ y un valor de 5 (40 \%), mientras que para los de GENP el nivel de interés decae un poco pero sigue siendo muy notable para todos, puesto que el $80 \%$ le asigna un valor de 4 .

Sobre la carga horaria existe una mayor disparidad de opiniones, siendo considerada de tipo moderado por los de primero (que la valoran mayoritariamente con un 3), mientras que para los de GENP supone un esfuerzo mayor, al estar situada fuera del horario habitual de la asignatura, como ya se ha comentado.

Dos aspectos importantes que revela la encuesta son los relacionados a la organización y coordinación entre ellos, igualmente bien valoradas, de forma que la puntuación asignada es el 4 o el 5, conjuntamente, para más de un $70 \%$ del alumnado, sin diferencias importantes para la Física y GENP.

Con el segundo bloque de preguntas se quiere determinar las sensaciones que han percibido los estudiantes al participar en FOREXPO. Este bloque tenía los siguientes items: exigencia por la dificultad del taller, nerviosismo por tener que hablar en público, sensación de liderazgo ante los asistentes, emoción, empatía hacia los asistentes y presencia de profesores. Cada item debía ser valorado igualmente de 1 a 5 . Los resultados de las respuestas facilitadas a estas cuestiones se muestran a continuación:

La exigencia académica les parece en general de bajo nivel o asequible a todos los universitarios, lo cual podría indicar, para los de primero, que, pese a tener poca base técnica, se empoderan y adquieren autoestima.

Para el nerviosismo por hablar en público, emoción y empatía hacia los asistentes a FOREXPO la dispersión de valoraciones es amplia, lo cual denotaría que se trata de aspectos 


\section{CONGRESO IBÉRICO DE AGROINGENIERÍA \\ X CONGRESSO IBÉRICO DE AGROENGENHARIA \\ 3 - 6 septiembre 2019, Huesca - España}

notablemente subjetivos. Mientras que para el liderazgo, al tratarse de algo observable objetivamente (por cuanto el alumno que explica se transforma en protagonista al ejercer learning by teaching), encontramos predominio claro del valor 4 , elegido por casi un $50 \%$ del alumnado de ambas asignaturas.

En cuanto a la presencia de los profesores, también es bien valorada, con 4 y 5 mayoritariamente, lo cual muestra una ausencia de antagonismo entre alumno-profesor y es prueba de confianza, pese a que el hecho de hablar en público pudiera parecer, a priori, una prueba dura que hiciera desear la ausencia de profesores.

Con el tercer bloque de preguntas se desea conocer en qué medida consideran los estudiantes del Grado que FOREXPO les aporta algo positivo de cara a su formación. Las cuestiones concretas que se les plantea valorar son las siguientes: la soltura a la hora de hablar en público, la mejora de la autoestima, la comprensión de partes de la asignatura expuesta, la mejora del interés por la asignatura, el acercamiento al profesor/a responsable, el conocimiento de los compañeros, la empatía hacia los demás y el grado de autonomía a la hora de trabajar. Al igual que en los dos bloques anteriores, las respuestas proporcionadas debían otorgar valores entre 1 y 5. Los resultados más destacados sobre estos aspectos se comentan a continuación:

Este quizás sea el bloque más relevante, por la valoración que hacen los universitarios sobre lo que supone FOREXPO a su formación personal. En general destaca que la valoración es mayoritariamente de 4 y 5 en todos los casos salvo en el acercamiento al profesor responsable, tal vez porque ya la relación es muy cercana y no se necesita una vivencia como FOREXPO para mejorar dicha relación, lo cual sería muy positivo. En general la relación es cercana y cordial y este resultado es esperable.

Also similar ocurre para la relación entre compañeros, puesto que al ser grupos pequeños suelen conocerse bastante bien y comprenden, por ende, lo que un compañero puede estar viviendo en FOREXPO.

En el cuarto bloque se les pide a los estudiantes que valoren las siguientes afirmaciones entre 1 y 5: "Enseñando se aprende más", "Con FOREXPO aprendo de mis compañeros" y "Con FOREXPO aprendo de los niños que nos visitan". Los resultados para estas afirmaciones han sido las siguientes:

El lema de Forexpo parece ser que es perfectamente asimilado y muy bien valorado por los universitarios, puesto que más del $90 \%$ le asigna un 4 o un 5 .

Además una gran mayoría reconoce que realiza aprendizaje a partir de sus compañeros y, en menor medida, de los niños.

Para finalizar la encuesta, se les preguntaba si la experiencia vivida en FOREXPO les anima a participar en otras actividades que se celebran habitualmente en los últimos años en el Centro Universitario de Plasencia como "La noche de los investigadores" o el "Desayuna con la ciencia". El resultado de sus respuestas ha sido el siguiente:

Entre los alumnos de la optativa de GENP el $100 \%$ desearía participar en iniciativas similares, mientras que solo el $40 \%$ de los de primero se animaría a estas. Puede deberse al carácter de educación medioambiental que pose GENP, lo cual hace que quienes la cursen ya contemplen como una actividad muy interesante la sensibilización y educación mediante actividades semejantes a FOREXPO.

\subsection{Encuestas de los profesores visitantes de los Centros de Educación Primaria}

Al igual que en el caso anterior, la encuesta a los profesores visitantes se estructuró en bloques temáticos, de modo que a las cuestiones planteadas en ellos en algunos casos debían hacerse valoraciones entre 1 y 5 con el mismo significado que se explicó anteriormente. Vamos a ir desgranando a continuación las reflexiones efectuadas por el profesorado de educación primaria a las cuestiones que se les planteó. 
En primer lugar se les preguntaba a los docentes si conocían la existencia de este tipo de actividades en el Centro Universitario de Plasencia. Ante esta cuestión, la respuesta facilitada fue que 4 de los 5 profesores que rellenaron la encuesta desconocían que en este Centro se desarrollaran este tipo de actividades, mientras que sólo uno de ellos afirmó conocerlas. Este resultado debe servir de reflexión al profesorado del Centro Universitario para el futuro, de modo que cuando se vayan a celebrar eventos de estas características se dé la oportuna difusión en los restantes Centros educativos de Plasencia y del norte de la provincia de Cáceres como forma de atraer a mayor número de visitantes a los mismos.

El segundo bloque de preguntas se elaboró para conocer la vía por la que se habían enterado de la actividad de FOREXPO, de modo que un encuestado respondió que se enteró porque contactaron directamente con él desde el Centro Universitario de Plasencia, mientras que los cuatro restantes contestaron que se enteraron a través del personal de su Centro de trabajo. El hecho de que algunos profesores del Centro Universitario tengan en su círculo familiar o de amistades a docentes de otros centros facilita el contacto directo con ellos, vía que debe seguir explotándose en la medida de lo posible. En cualquier caso, también es destacable que la información ofrecida a los Centros fue debidamente canalizada por el personal que trabaja en ellos para informar debidamente de este tipo de actividades a los docentes que potencialmente pudieran estar más directamente interesados en que sus estudiantes acudan a los mismos.

En el tercer bloque se deseaba recoger las impresiones de los docentes a raíz de su experiencia al asistir a FOREXPO. El 80\% de los encuestados (4 en total) afirmaron que les resultó totalmente novedosa la experiencia mientras que al $20 \%$ restante ( 1 persona) no les pareció muy novedosa, otorgándole una puntuación de 3 . Con respecto al interés personal en esta experiencia el $80 \%$ tenían muchísimo interés (valoración de 5), mientras que el 20\% tenía un elevado interés (4 puntos). En cuanto al interés que en su opinión esta actividad debía revestir para sus estudiantes, la valoración realizada fue unánime (5 puntos), considerando por lo tanto que FOREXPO posee el máximo interés para sus estudiantes, algo que reconforta a los docentes responsables de las asignaturas implicadas pues ése era un objetivo primordial para seguir ofertando esta actividad en el futuro. Al preguntarles por la duración de la actividad, nuevamente el $80 \%$ de los encuestados contestaron que les pareció muy adecuada la duración de la actividad (5 puntos), mientras que el $20 \%$ opinó que ni bien ni mal (3 puntos). A la última cuestión planteada en este bloque, sobre la organización de la actividad, el $80 \%$ estaba totalmente de acuerdo en cómo se había organizado ésta, mientras que el $20 \%$ opinó que estaba de acuerdo.

En el cuarto bloque de preguntas se desea conocer las sensaciones que los profesores visitantes han percibido en sus estudiantes al asistir a FOREXPO, teniendo que valorar de 1 a 5 las diferentes cuestiones planteadas. De las respuestas facilitadas se constató que de manera unánime los docentes percibieron que tanto la ilusión despertada en los escolares como el interés despertado por los talleres fueron máximos, del mismo modo que hubo unanimidad, pero ésta en sentido contrario (valoración de 1) en que los escolares en ningún momento se sintieron cohibidos por la presencia de los profesores, algo esperado por otro lado, mientras que el $80 \%$ de los encuestados respondieron que no notaron excesivo nerviosismo en ellos ( 2 puntos) frente al $20 \%$ que percibió un nerviosismo normal ( 3 puntos) en los días u horas previos al desarrollo de esta actividad. Además, también coincidieron ( $80 \%$ con valoración de 5 frente al $20 \%$ con valoración de 4) en que esta actividad puede contribuir a despertar el interés de los estudiantes por la ciencia, valoración muy positiva en este caso, y los mismos porcentajes y valores se alcanzaron para estimar la empatía mostrada por los escolares hacia los estudiantes del Grado en IFMN que se encargaban de explicarles los diferentes talleres.

En el quinto bloque se desea saber en qué medida los docentes visitantes consideran que FOREXPO puede contribuir a mejorar la formación de sus estudiantes. El $80 \%$ asignó una valoración de 5 a esta cuestión, mientras que el 20\% restantes le otorgó una puntuación de 4, deduciéndose por lo tanto que los docentes ven en FOREXPO una buena oportunidad para que sus estudiantes se motiven más por los estudios y mejoren así su formación. Esta misma 


\section{CONGRESO IBÉRICO DE AGROINGENIERÍA \\ X CONGRESSO IBÉRICO DE AGROENGENHARIA \\ 3 - 6 septiembre 2019, Huesca - España}

valoración y estos mismos porcentajes fueron los que se registraron cuando se les preguntó a los docentes en qué medida FOREXPO puede contribuir a mejorar el interés por la ciencia en sus alumnos, deduciéndose en este caso también que tienen depositadas grandes esperanzas en que esta y otras actividades de este tipo puedan contribuir a dicha mejora.

En el sexto bloque se les preguntó en qué medida conocían otras actividades que se desarrollan habitualmente en el Centro Universitario de Plasencia en los últimos años y a los que asisten asiduamente escolares llegados desde distintos lugares de la provincia de Cáceres, respondiendo el $80 \%$ que conocían tanto "La noche de los investigadores" como las "jornadas de puertas abiertas", frente al $20 \%$ que no estaba al corriente de tales iniciativas. A diferencia de los anteriores, todos los encuestados afirmaron conocer el "Desayuna con la ciencia".

Para finalizar, todos los encuestados respondieron que estarían encantados de repetir su visita a FOREXPO en años venideros, lo que anima fervientemente a los organizadores a continuar con esta propuesta que ha resultado ser tan bien valorada tanto por los docentes como por los escolares.

\section{Conclusiones}

La iniciativa de FOREXPO resulta ser muy bien valorada, tanto a nivel cualitativo (a través de felicitaciones y comentarios verbales que habitualmente se reciben), como a nivel cuantitativo, según muestran los resultados de las encuestas del presente estudio.

Por una parte los alumnos consideran que es una iniciativa altamente novedosa y muy interesante, aun para los que ya la conocieron en primer curso y ahora no tiene el mismo factor sorpresa.

El factor emocional es muy importante, aun cuando los alumnos más veteranos ya conocen la experiencia desde primero. Esto garantiza el éxito de esta iniciativa de learning by teaching, puesto que el aprendizaje significativo lo es tanto más cuanta mayor sea la emoción con la que el alumno desarrolla una actividad de aprendizaje, siendo un método de anclaje de conocimientos muy efectivo la implicación emocional.

En la parte más objetiva, los alumnos consideran que se trata de una buena herramienta para motivarles puesto que aumenta el interés por la asignatura, así como la comprensión de algunas partes de la misma. Del mismo modo la consideran muy útil para mejorar la autoestima y la capacidad de hablar ante un público no especializado.

En cuanto a las relaciones puestas en juego, éstas forman parte muy importante también de la actividad y aprendizaje emocional de esta experiencia, no solo entre compañeros estudiantes, sino también con los niños y con los profesores (tutores y visitantes).

Podemos añadir que, globalmente, FOREXPO resulta una experiencia muy enriquecedora desde el punto de vista de los aprendizajes adquiridos y de las competencias transversales y la vivencia emocional experimentada, que contribuye a anclar dichos aprendizajes.

\section{Referencias}

1. González J., Wagenaar R. Tuning educational structures in Europe. Universidad de Deusto. Bilbao, España. 2003.

2. García E., Benítez R., Villar J.R., López M.L., González R., Moya M. The use of whatsapp for improving the student attention from the tutorial action plan. Proceedings of the 9th Annual Conference of Education, Research and Innovation (ICERI 2016). IATED. Sevilla, España, 14-16 noviembre 2016, pp. 2660-2665.

3. Moya M., García M.E., Hernández J., Montero M.J., Martínez R., López M.L., Benítez R., Villar J.R. Education from affection, also at the University? Proceedings of the 8th Annual Conference of Education, Research and Innovation (ICERI 2015). IATED. Sevilla, España, 16-18 noviembre 2015, pp. 1539-1545. 


\section{CONGRESO IBÉRICO DE AGROINGENIERÍA \\ $X$ CONGRESSO IBÉRICO DE AGROENGENHARIA \\ 3 - 6 septiembre 2019, Huesca - España}

4. Benítez R., García E., Montero M.J., Martínez R., Moya M., Solla A., Hernández J. Learning by teaching within a University-Society interaction framework. Proceedings of the 6th Annual Conference of Education, Research and Innovation (ICERI 2014). IATED. Sevilla, España, 17-19 noviembre 2014, pp. 1124-1130.

5. Moya M., García E., Villar J.R., López L., Benítez R. Skills required by companies from Forest Engineers. Experiences from the University-Alumni-Company relation at the University of Extremadura. Proceedings of the 6th Annual Conference of Education, Research and Innovation (ICERI 2014). IATED. Sevilla, España, 17-19 noviembre 2014, pp. 1207-1214.

6. Cherniss C. Emotional intelligence: what it is and why it matters. Annual Meeting of the Society for Industrial and Organizational Psychology, New Orleans, Los Ángeles, Estados Unidos de América, 15 de abril de 2000.

7. Goleman D. Emotional intelligence: why it can matter more than IQ. Bloomsbury Publishing Ltd. Reino Unido de Gran Bretaña. 1996, 367 pp.

8. Goleman D. An EI-based theory of performance. En Cherniss C., Goleman D. (eds.). The emotionally intelligence workplace. Jossey-Bass, San Francisco, Estados Unidos de América. 2001, pp. 27-45.

9. Fook C.Y. Best practices of teaching in higher education in United States: a case study. Procedia-Social and Behavioral Sciences. 2012, 46, 4817-4821.

10. Olsen P.B., Pedersen K. Working with your Supervisor. En Olsen P.B., Pedersen K. (eds.). ProblemOriented Project Work- A Workbook. Roskilde University Press. Denmark. 2005, 123-134.

11. Campo L. El aprendizaje servicio en la universidad como propuesta pedagógica. En Martínez M. (ed.). Aprendizaje, servicio y responsabilidad social de las universidades. Octaedro-ICE-UB. Barcelona, España. 2010, 81-91.

12. Gartner A., Kohler M.C., Riessman F. Children teach children: learning by teaching. Harper \& Row. Nueva York, Estados Unidos de América. 1971.

13. Blazar D., Kraft M.A. Teacher and teaching effects on students' attitudes and behaviors. Educational Evaluation and Policy Analysis. 2017, 39(1), 146-170.

14. Fiorella L., Mayer R.E. The relative benefits of learning by teaching and teaching expectancy. Contemporary Educatonal Psychology. 2013, 38, 281-288.

15. Fiorella L., Mayer R.E. Role of expectations and explanations in learning by teaching. Contemporary Educatonal Psychology. 2014, 39, 75-85.

16. Park S., Kim C. Boosting learning-by teaching in virtual tutoring. Computers and Education. 2015, 82, 129-140.

17. Brevik L., Gunnulfsen A.E., Renzulli J.S. Student teachers' practice and experience with differentiated instructions for students with higher learning potential. Teaching and Teacher Education. 2018, 71, 3445.

18. Denancé V., Somat A. Learning by explaining: impacts of explanations on the development of a competence. Revue européene de psychologie appliquée. 2015, 65, 307-315. 